Proceedings of the 2003 IEEE/ASME

International Conference on

Advanced Intelligent Mechatronics (AIM 2003)

\title{
Kinematics Optimization for High Positioning Accuracy of a 4-DOF Parallel Manipulator for Semiconductor Applications
}

\author{
Jacob W.F. Cheung ${ }^{1,2}$ \\ ${ }^{1}$ Research and Development Department, \\ ASM Assembly Automation Ltd., 5/F Watson Centre, \\ 16 Kung Yip Street, Kwai Chung, New Territories, \\ Hong Kong. \\ E-mail: jacob.cheung@asmpt.com
}

\author{
Y.S. Hung ${ }^{2}$ \\ ${ }^{2}$ Department of Electrical and Electronic Engineering, \\ The University of Hong Kong, Pokfulam Road, \\ Hong Kong. \\ E-mail:yshung@eee.hku.hk
}

\begin{abstract}
The end-effector kinematics error of a manipulator is caused by variations in machining accuracy of linkages. In most manipulators, the structural design results in an error magnification from the linkage variation to the end-effector position. In this paper, an optimization approach for suppressing kinematics error magnification is considered for a 4-DOF parallel manipulator. Two objective functions are developed for characterizing the error magnification effect. The kinematic parameters are then determined by minimizing these objective functions. It is shown that the proposed approach can reduce the error magnification to such a degree that the magnification factor is reduced to close to one. This means that the end-effector kinematics error can be made to match the error tolerence allowed in the manufacturing of the linkages.
\end{abstract}

Keywords

Parallel Manipulator, Kinematics, Optimization

\section{INTRODUCTION}

Kinematics optimization is essential for achieving high positioning accuracy and compactness of a multiple-DOF parallel manipulator. The manipulator dexterity, operational workspace and structural stiffness have been used as the performance index for most of the existing optimization algorithms. A global performance index has been proposed by Gosselin and Angeles [5] to minimize the kinematics error due to the transformation between joint and Cartesian space. The global performance index has been modified by Tsai and Joshi [8] and LeguayDurand et al. [6] to optimize the stiffness and the dexterity of the manipulator workspace. The global dexterity index was recently proposed by Gallant and Boudreau [4] to optimize the workspace of the planar parallel manipulators. The quasi-Newton optimization algorithm was proposed by Carretero et al. [2] to minimize the parasitic motion of a 3dof spatial manipulator. A nonlinear programming method had been proposed by Bhattacharya et al. [1] to maximize the rigidity of the Stewart platform over the desired workspace.
A method for optimal kinematics design of the Hexaslide type parallel manipulator was recently proposed by Ryu and Cha [7]. The error kinematics model of the manipulator was derived and an objective function defined to be the product of the singular values of the error model was used for constrained optimization. The weakness of this approach is that the minimization of the singular values product does not ensure that the kinematics error is reduced in all directions of the workspace.

In high precision positioning mechanisms, end-effector accuracy and repeatability are essential in assuring the product quality. Furthermore, the size of the mechanism is required to be compact with respect to the workspace. Under these constraints, the structural design of the manipulator may cause the manufacturing variations of the mechanical linkages to be magnified at the position of the end-effector. The accuracy of the linkages is constrained by the manufacturing capability of the machines. To overcome the problem of error magnification, optimization approaches are developed to determine the kinematic parameters so as to minimize the kinematics error within a prescribed reachable workspace volume.

In this paper, a kinematics optimization approach for high precision positioning accuracy of manipulators is considered. Kinematics optimization methods based on two new objective functions are developed for a 4-DOF parallel manipulator developed in [3]. The proposed objective functions yield better kinematic parameters (with respect to the suppression of kinematic error magnification) compared with that obtained using the method of [7]. The paper is organized as follows. In section 2, the error magnification matrix for a 4-DOF parallel mechanism under consideration is derived. Two objective functions for minimization of the end-effector kinematics error are developed in section 3. The optimization process and the constraints of the design variables are described in Section 4. In Section 5, the results of the optimization are given together with the statistical analysis of the kinematics error distribution of the end-effector in an operational workspace. 


\section{PROBLEM FORMULATION}

In a semiconductor packaging system, the positioning mechanism is usually required to provide 4-DOF motion, where 3 of these motions are translations in the $\mathrm{XYZ}$ space, and the remaining DOF is a rotational motion against the Z-axis.

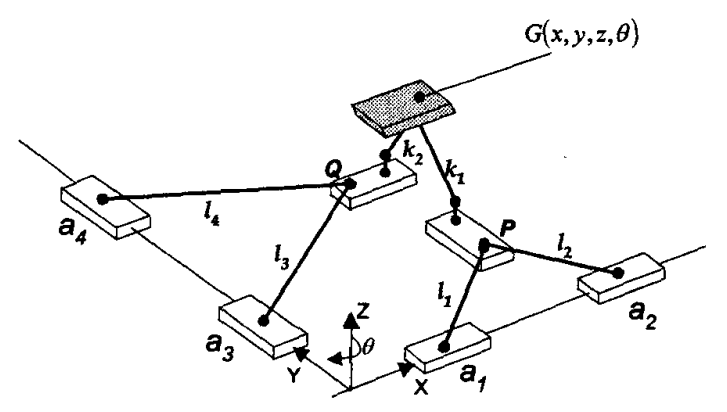

Figure 1. Kinematics design of the 4-DOF parallel manipulator.

Figure 1 shows the kinematics design of the 4-DOF parallel manipulator proposed in [3]. The manipulator consists of 3DOF of translation in XYZ space and 1-DOF in rotation against the $Z$-axis. The linear miniature servomotor pairs $a_{1} a_{2}$ and $a_{3} a_{4}$ are coupled to two 2-DOF planar mechanisms on the $\mathrm{XY}$ plane. A triangular structure is constructed on the two planar mechanisms with motion restricted to a vertical plane.

Let the locations of the four linear actuators be $a_{n}$ ( $n=$ $1,2,3,4)$ satisfying the conditions,

$$
\begin{aligned}
& 0<a_{1 \min } \leq a_{1} \leq a_{1 \max }<a_{2 \min } \\
& a_{2 \min } \leq a_{2} \leq a_{2 \max } \\
& 0<a_{3 \min } \leq a_{3} \leq a_{3 \max }<a_{4 \min } \\
& a_{4 \min } \leq a_{4} \leq a_{4 \max }
\end{aligned}
$$

In the case when $l_{1}=l_{2}=l_{3}=l_{4}=l$ and $k_{1}=k_{2}=k$, the coordinates of the points $P\left(x_{1}, y_{1}\right)$ and $Q\left(x_{2}, y_{2}\right)$ are related to the actuator positions by:

$$
\begin{aligned}
& x_{1}=\frac{1}{2}\left(a_{1}+a_{2}\right) \\
& y_{1}=\sqrt{l^{2}-\frac{1}{4}\left(a_{2}-a_{1}\right)^{2}} \\
& x_{2}=\sqrt{l^{2}-\frac{1}{4}\left(a_{4}-a_{3}\right)^{2}} \\
& y_{2}=\frac{1}{2}\left(a_{3}+a_{4}\right)
\end{aligned}
$$

The coordinates of the end-effector, denoted $G(x, y, z, \theta)$, can be related to the coordinates of $P\left(x_{1}, y_{1}\right)$ and $Q\left(x_{2}, y_{2}\right)$ as:

$$
\begin{aligned}
& x=\frac{1}{2}\left(x_{1}+x_{2}\right) \\
& y=\frac{1}{2}\left(y_{1}+y_{2}\right) \\
& z=\sqrt{k^{2}-\frac{\left(x_{2}-x_{1}\right)^{2}+\left(y_{2}-y_{1}\right)^{2}}{4}} \\
& \theta=\tan ^{-1}\left(\frac{y_{2}-y_{1}}{x_{2}-x_{1}}\right)
\end{aligned}
$$

Suppose the mechanical linkages have manufacturing variations:

$$
\delta L=\left[\begin{array}{llllll}
\delta l_{1} & \delta l_{2} & \delta l_{3} & \delta l_{4} & \delta k_{1} & \delta k_{2}
\end{array}\right]^{T}
$$

These variations lead to a kinematics error of the endeffector of the manipulator:

$$
\delta E=\left[\begin{array}{llll}
\delta x & \delta y & \delta z & \delta \theta
\end{array}\right]^{T}
$$

$\delta E$ can be related to $\delta L$ by:

$$
\delta E=E_{M} \cdot \delta L
$$

where

$$
\begin{aligned}
& E_{M}=\left[\begin{array}{cccccc}
e_{11} & -e_{11} & e_{12} & e_{12} & e_{13} & -e_{13} \\
e_{21} & e_{21} & e_{22} & -e_{22} & e_{23} & -e_{23} \\
e_{31} & e_{32} & e_{33} & e_{34} & e_{35} & e_{35} \\
e_{41} & e_{42} & e_{43} & e_{44} & 0 & 0
\end{array}\right] \\
& e_{11}=\frac{l}{2\left(a_{2}-a_{1}\right)} \\
& e_{12}=\frac{l}{4 x_{2}} \\
& e_{13}=\frac{k \cos \theta}{\sqrt{\left(y_{2}-y_{1}\right)^{2}+\left(x_{2}-x_{1}\right)^{2}}} \\
& e_{21}=\frac{l}{4 y_{1}} \\
& e_{22}=\frac{l}{2\left(a_{4}-a_{3}\right)} \\
& e_{23}=\frac{k \sin \theta}{\sqrt{\left(y_{2}-y_{1}\right)^{2}+\left(x_{2}-x_{1}\right)^{2}}} \\
& e_{31}=\frac{\left(x_{1}-x_{2}\right)}{4 z\left(a_{2}-a_{1}\right)}+\frac{\left(y_{1}-y_{2}\right)}{8 z \sqrt{l^{2}-\frac{\left(a_{2}-a_{1}\right)^{2}}{4}}} \\
& e_{32}=\frac{-\left(x_{1}-x_{2}\right) l}{4 z\left(a_{2}-a_{1}\right)}+\frac{\left(y_{1}-y_{2}\right)}{8 z \sqrt{l^{2}-\frac{\left(a_{2}-a_{1}\right)^{2}}{4}}}
\end{aligned}
$$




$$
\begin{aligned}
& e_{33}=\frac{\left(x_{2}-x_{1}\right) l}{8 z \sqrt{l^{2}-\frac{\left(a_{4}-a_{3}\right)^{2}}{4}}}+\frac{\left(y_{2}-y_{1}\right) l}{4 z\left(a_{4}-a_{3}\right)} \\
& e_{34}=\frac{\left(x_{2}-x_{1}\right) l}{8 z \sqrt{l^{2}-\frac{\left(a_{4}-a_{3}\right)^{2}}{4}}}-\frac{\left(y_{2}-y_{1}\right) l}{4 z\left(a_{4}-a_{3}\right)} \\
& e_{35}=\frac{k}{2 \sqrt{k^{2}-\frac{\left(y_{2}-y_{1}\right)^{2}+\left(x_{2}-x_{1}\right)^{2}}{4}}} \\
& \left.e_{41}=\frac{l}{\left[\left(x_{2}-x_{1}\right)^{2}+\left(y_{2}-y_{1}\right)^{2}\right.}\right]\left[\frac{\left(y_{2}-y_{1}\right)}{\left(a_{2}-a_{1}\right)}-\frac{\left(x_{2}-x_{1}\right)}{2 \sqrt{l^{2}-\frac{\left(a_{2}-a_{1}\right)^{2}}{4}}}\right] \\
& \left.e_{42}=\frac{l}{\left[\left(x_{2}-x_{1}\right)^{2}+\left(y_{2}-y_{1}\right)^{2}\right.}\right]\left[\frac{-\left(y_{2}-y_{1}\right)}{\left(a_{2}-a_{1}\right)}-\frac{\left(x_{2}-x_{1}\right)}{2 \sqrt{l^{2}-\frac{\left(a_{2}-a_{1}\right)^{2}}{4}}}\right] \\
& \left.e_{43}=\frac{l}{\left(\left(x_{2}-x_{1}\right)^{2}+\left(y_{2}-y_{1}\right)^{2}\right.}\right]\left[\frac{-\left(y_{2}-y_{1}\right)}{2 \sqrt{l^{2}-\frac{\left(a_{4}-a_{3}\right)^{2}}{4}}}+\frac{\left(x_{2}-x_{1}\right)}{\left(a_{4}-a_{3}\right)}\right] \\
& \left.e_{44}=\frac{l}{\left[\left(x_{2}-x_{1}\right)^{2}+\left(y_{2}-y_{1}\right)^{2}\right.}\right]\left[\frac{-\left(y_{2}-y_{1}\right)}{2 \sqrt{l^{2}-\frac{\left(a_{4}-a_{3}\right)^{2}}{4}}}-\frac{\left(x_{2}-x_{1}\right)}{\left(a_{4}-a_{3}\right)}\right]
\end{aligned}
$$

From (11), we see that the Jacobian matrix $E_{M}$ defines how the variations of the mechanical linkages is transformed into end-effector kinematics error in the coordinate $G(x, y$, $z, \theta$ ). In most manipulators, $E_{M}$ has a magnification effect so that $\delta L$ is amplified to become larger errors at the endeffector. Since the size of $\delta L$ is prescribed by the machining capability, we can only improve the kinematics positioning accuracy by making $E_{M}$ as 'small' as possible. In the next section, we will consider how the parameters of the linkages can be optimized to ensure that $E_{M}$ and hence $\delta E$ is small.

\section{DESIGN OF OBJECTIVE FUNCTIONS}

Let $E_{M}$ be factorized using Singular Value Decomposition as:

$$
E_{M}=U S V^{T}
$$

where $U=\left[u_{1} \ldots u_{4}\right]$ and $V=\left[v_{1} \ldots v_{4}\right]$ are (part of) unitary matrices, and $S=\operatorname{diag}\left(\sigma_{1} \cdots \sigma_{4}\right)$ is a diagonal matrix with the singular values $\sigma_{i}(i=1,2,3,4)$ satisfying $\sigma_{1} \geq \ldots \geq \sigma_{4} \geq$ 0 . The singular value decomposition can be regarded as a principal component analysis of the error magnification matrix $E_{M}$, with the singular values representing the error amplification along different principal directions defined by the singular vectors. From (28), since $U$ and $V$ are unitary, the error magnification matrix can be reduced by minimizing the singular values of $S$.

In [7], it is proposed that the product of the singular values, (i.e. $\Pi \sigma_{i}$ ) is minimized over a workspace volume. The drawback of using the product of the singular values as an objective function in the minimization is that very little control can be exercised over the magnitude of individual singular values. For example, the product is suppressed by making only one particular singular value small while leaving the others unchecked. This means that error amplification is reduced only along one direction of the workspace corresponding to the suppressed singular value. It would be more desirable to reduce all singular values of $E_{M}$ as a whole so that error amplification is suppressed uniformly in all directions of the workspace.

We note that the Frobenius norm of $E_{M}$ is related to the singular values by:

$$
\left\|E_{M}\right\|_{F}^{2}=\|S\|_{F}^{2}=\sum_{i=1}^{4} \sigma_{i}^{2}
$$

Since all the singular values are bounded above by $\left\|E_{M}\right\|_{F}$, the minimization of the Frobenius norm of $E_{M}$ ensures that all singular values are bounded below this value. We will next propose the minimization of two objective functions for the purpose of suppressing the end-effector kinematics error over an operational workspace $W$.

\section{A. Minimizing the Integral of $\sum_{i=1}^{4} \sigma_{i}^{2}$ over $W$}

Since $E_{M}$ depends not only on the kinematic parameters $(l, k)$, but also on the position $(x, y, z, \theta)$ of the endeffector, it is necessary to ensure that $E_{M}$ remains small over a chosen operational workspace $W$. For this purpose, we define an objective function to be the integral of $\left\|E_{M}\right\|_{F}$ over the operational workspace:

$$
f_{1}(l, k)=\frac{1}{W} \int_{W} \sum_{i=1}^{4} \sigma_{i}^{2} d w
$$

The kinematic parameters $l$ and $k$ are then determined by solving the minimization problem:

$$
\min _{l, k} f_{1}(l, k)
$$

This means that $l$ and $k$ are optimized to reduce the average (or typical) error amplification over $W$. The accuracy of the system is however often expressed in terms of the worst-case error. For this reason, we will consider a second objective function for reducing the worst-case kinematic errors magnification. 


\section{B. Minimizing the Maximum of $\left\|E_{M}\right\|_{F}^{2}$ over $W$}

The objective function for the minimization of the worstcase kinematics error magnification over the operational workspace is defined as:

$$
f_{2}(l, k)=\max _{(x, y, z, \theta) \in W}\left(\sum_{i=1}^{4} \sigma_{i}^{2}\right)
$$

Let $\left(l^{*}, k^{*}\right)$ be the solution to the minimization problem:

$$
\min _{l, k} f_{2}(l, k)
$$

$f_{2}\left(l^{*}, k^{*}\right)$ can be used to obtain a hard upper bound on the end-effector kinematics error over the entire operational workspace $W$, which can then serve as a specification of the system in the sense that all points within $W$ will have an error no larger than this value. The minimization of $f_{2}(l, k)$ thus have the meaning of designing the system for the best specification.

\section{OPTIMIZATION PROCESS}

The objective of the optimization algorithm is to minimize the kinematics error of the end-effector of the manipulator in the operational workspace. A cubic volume in the centre of the manipulator workspace is selected to form the operational workspace. The size of the operational workspace is equal to $(\Delta x, \Delta y, \Delta z)=(15 \mathrm{~mm}, 15 \mathrm{~mm}, 2 \mathrm{~mm})$. The operational workspace is divided into 432 grid points for evaluating the numerical integration in the case of the objective function $f_{1}$ and for taking the maximum in the case of $f_{2}$.

The design variables of the optimization are the linkage $l$ of the two 2-DOF planar mechanism and the linkage $k$ of the triangular structure as shown in fig. 1 . The maximum error budget of the length of both $l$ and $k$ is set at $1 \mu \mathrm{m}$. The length of $l$ and $k$ are constrained within the linkage boundaries to avoid excessive geometrical error magnification. The boundary singularities of the manipulator can be determined by the geometrical characteristic equations:

$$
\begin{aligned}
& k=\left(y_{2}-y_{1}\right) / 2 \text { and } x_{1}=x_{2} \\
& l=\left(a_{2}-a_{1}\right) / 2 \text { or } l=\left(a_{4}-a_{3}\right) / 2
\end{aligned}
$$

These boundary singularities are avoided in the optimization process by constraining the length of $l$ and $k$.

A constrained nonlinear optimization method is used for the minimization of the kinematics error of the endeffector. The Sequential Quadratic Programming (SQP) method is used for minimizing the value of the objective function. In each iteration, the Hessian matrix of the Lagrangian function is calculated using the BFGS method.
The projected gradient is calculated to determine the step size of the change of the design variables. Finally, the design variables are modified for the next iteration. The iteration is terminated when the difference of the objective function derivative in two iterations is less than a certain minimum tolerance.

\section{OPTIMIZATION RESULTS AND ANALYSIS}

A given set of dimensional parameters of the linkage is used to generate the surface of the proposed objective functions. A plot of the surfaces of objective functions $f_{1}(l, k)$ and $f_{2}(l, k)$ over the feasible region of the constrained parameters $l$ and $k$ are shown in Figures 2 and 3 respectively. In these surfaces, no local minimum of the objective functions exists within the feasible region. This means that under the constraints imposed on the kinematic parameters, the optimal solution lies on the boundary of the feasible region.

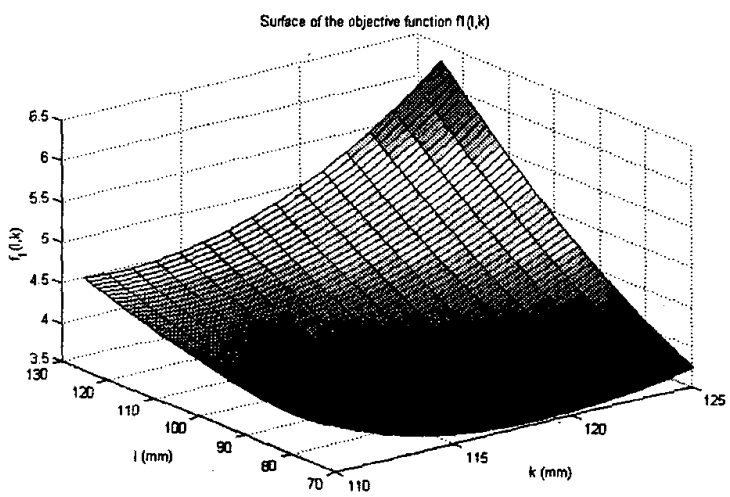

Figure 2. Surface of the objective function $f_{1}(I, k)$.

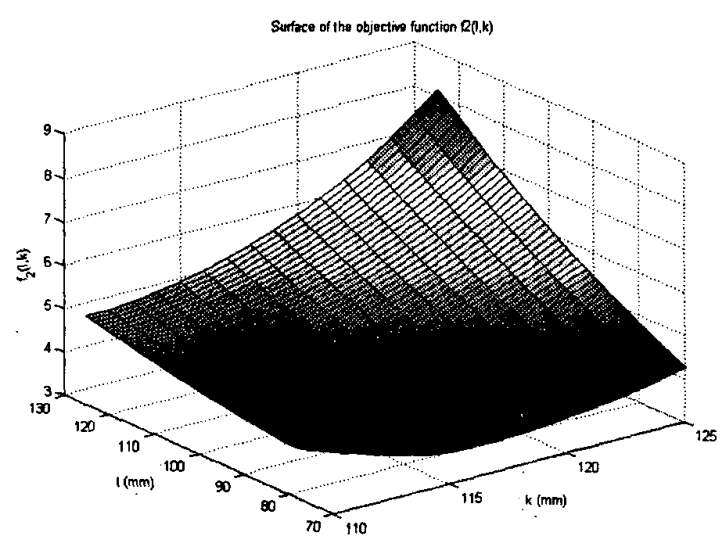

Figure 3. Surface of the objective function $f_{2}(I, k)$.

Figures 4 and 5 show a plot of the values of the objective functions $f_{1}(l, k)$ and $f_{2}(l, k)$ respectively at the 432 individual test points in the operational workspace $W$. 
These plots show how the objective functions are scattered around the optimal value after optimization.

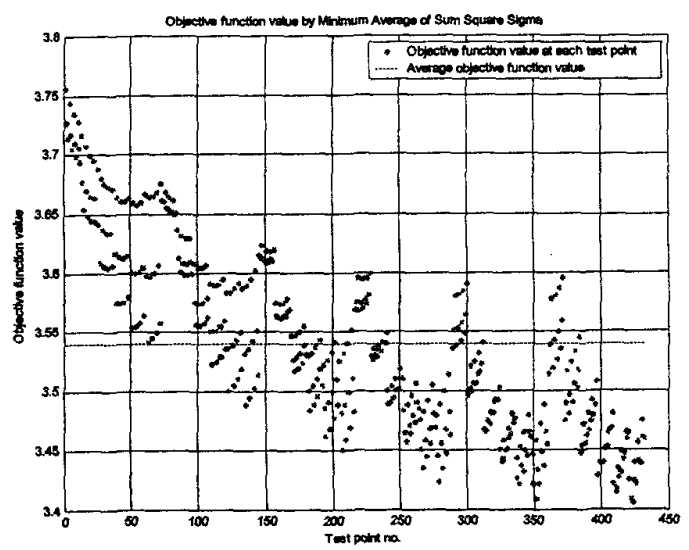

Figure 4. Values of the objective function $f_{1}(I, k)$ at the individual test points in the workspace $W$.

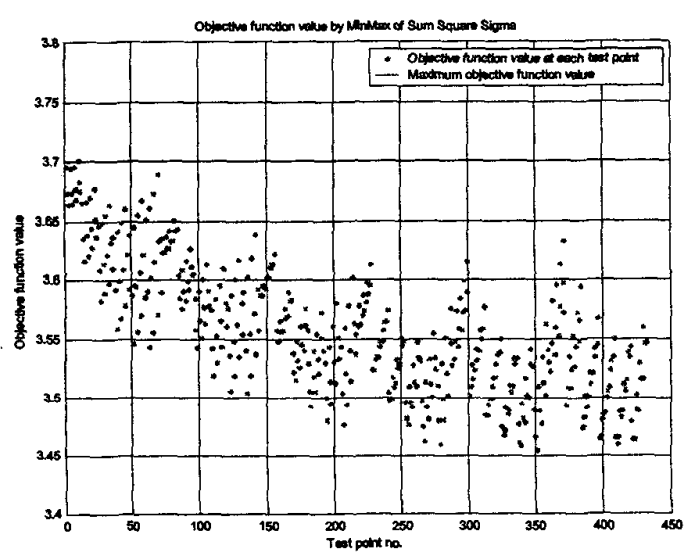

Figure 5. Values of the objective function $f_{2}(I, k)$ at the individual test points in the workspace $W$.

We observe from Figures 4 and 5 that although the optimal value of $f_{1} \quad(=3.535)$ is smaller than that of $f_{2}(=3.70)$, the latter represents a hard upper bound for all grid points in the workspace whereas $f_{1}$ can only be read as an average error. Furthermore, the range as well as the scattering of the points is slightly smaller in the case of the optimal solution for $f_{2}$.

The initial parameters of the linkages $l$ and $k$, and the optimal parameters for the objective functions $f_{1}$ and $f_{2}$ after optimization are shown in Table 1.
Table 1. Optimization results of the linkages and the objective functions of the manipulator.

\begin{tabular}{|l|c|c|c|}
\hline & $l(\mathrm{~mm})$ & $\begin{array}{c}k \\
(\mathrm{~mm})\end{array}$ & $\begin{array}{c}\text { Objective } \\
\text { function }\end{array}$ \\
\hline Initial parameters of $f_{1}(l, k)$ & 130 & 130 & 153.125 \\
\hline Initial parameters of $f_{2}(l, k)$ & 130 & 130 & 626.627 \\
\hline Optimal solution for $f_{1}(l, k)$ & 70 & 118.6 & 3.535 \\
\hline Optimal solution for $f_{2}(l, k)$ & 70 & 116.6 & 3.70 \\
\hline
\end{tabular}

Table 2. Statistical analysis of the initial kinematics positioning accuracy.

\begin{tabular}{|l|l|l|l|l|}
\hline & $\delta \mathrm{x}(\mu \mathrm{m})$ & $\delta \mathrm{y}(\mu \mathrm{m})$ & $\delta \mathrm{z}(\mu \mathrm{m})$ & $\delta \theta(\mathrm{mdeg})$ \\
\hline Minimum & 3.79 & 3.79 & -91.66 & -602.59 \\
\hline Maximum & 106.09 & 106.09 & -1.52 & 602.59 \\
\hline Average & 12.17 & 12.17 & -8.86 & 0 \\
\hline $\begin{array}{l}\text { Standard } \\
\text { deviation }\end{array}$ & 15.89 & 15.89 & 13.89 & 139.98 \\
\hline
\end{tabular}

From Table 2, we see that with the initial kinematic parameters, a variation of only $1 \mu \mathrm{m}$ in each of the linkage of $l$ and $k$, the average kinematics positioning error of the end-effector is magnified to $12 \mu \mathrm{m}$ in the XY plane and $8 \mu \mathrm{m}$ in the Z-axis.

The kinematics positioning error after optimization using the objective functions $f_{1}(l, k)$ and $f_{2}(l, k)$ are shown in Tables 3 and 4 respectively.

Table 3. Statistical analysis of the kinematics positioning accuracy optimized by $f_{1}$.

\begin{tabular}{|l|l|l|l|l|}
\hline & $\delta \mathrm{x}(\mu \mathrm{m})$ & $\delta \mathrm{y}(\mu \mathrm{m})$ & $\delta \mathrm{z}(\mu \mathrm{m})$ & $\delta \theta(\mathrm{mdeg})$ \\
\hline Minimum & 0.68 & 0.68 & 0.68 & -2.72 \\
\hline Maximum & 1.03 & 1.03 & 0.89 & 2.72 \\
\hline Average & 0.83 & 0.83 & 0.8 & 0 \\
\hline $\begin{array}{l}\text { Standard } \\
\text { deviation }\end{array}$ & 0.089 & 0.089 & 0.049 & 1.225 \\
\hline
\end{tabular}

Table 4. Statistical analysis of the kinematics positioning accuracy optimized by $f_{2}$.

\begin{tabular}{|l|l|l|l|l|}
\hline & $\delta \mathrm{x}(\mu \mathrm{m})$ & $\delta \mathrm{y}(\mu \mathrm{m})$ & $\delta \mathrm{z}(\mu \mathrm{m})$ & $\delta \theta(\mathrm{mdeg})$ \\
\hline Minimum & 0.65 & 0.65 & 0.73 & -2.47 \\
\hline Maximum & 0.96 & 0.96 & 0.9 & 2.47 \\
\hline Average & 0.78 & 0.78 & 0.83 & 0 \\
\hline $\begin{array}{l}\text { Standard } \\
\text { deviation }\end{array}$ & 0.079 & 0.079 & 0.042 & 1.147 \\
\hline
\end{tabular}

Tables 3 and 4 show that a significant reduction of the kinematics positioning error of the end-effector has been achieved by minimizing either $f_{1}(l, k)$ or $f_{2}(l, k)$. With the optimized solution, the kinematics error of the end-effector is of the same order of magnitude as the variation of the linkages $l$ and $k$. This is in contrast to an error magnification that is usually found in many mechanisms. Indeed, if we had used the initial parameters in the present mechanism, the variations in the linkages would result in a kinematics error of about an order of magnitude larger than the errors in the linkages. 


\section{CONCLUSIONS}

Although we have developed the optimization approach for reducing the kinematics error magnification specifically for the 4-DOF parallel manipulator of [3], the method described in this paper is applicable to other mechanisms. A significant improvement of the kinematics positioning accuracy demonstrates the capability of the proposed optimization method for high accuracy semiconductor packaging applications. The objective functions developed in this paper have been found to give better results compared with the objective function used in [7]. Furthermore, we have shown that the 4-DOF parallel manipulator, together with suitable choices of the kinematic parameters using the optimization approach developed in this paper, can achieve a high positioning accuracy which matches the error tolerence allowed in the manufacturing of the linkages. Hence, the results of this paper provide a justification for the structural design of the 4-DOF manipulator.

\section{ACKNOWLEDGMENTS}

This research project is funded and supported by ASM Assembly Automation Ltd. http://www.asmpacific.com. The authors gratefully acknowledge the technical help and advice received from Dr. P.C.K. Liu, Dr. G.P. Widdowson and Mr. S.K. Wong of ASM.

\section{REFERENCES}

[1] S. Bhattachaya, H. Hatwal and A. Ghosh, "On the optimum design of stewart platform type parallel manipulators", Robotica, Vol.13, pp.133-140, 1995.
[2] J.A. Carretero, R.P. Podhorodeski, M.A. Nahon and C.M. Gosselin, "Kinematics analysis and optimization of a new three degree-of-freedom spatial parallel manipulator", Journal of Mechanical Design, Vol.122, pp.17-24, 2000.

[3] Jacob W.F. Cheung, Y.S. Hung and G.P. Widdowson, "Design and analysis of a novel 4-dof parallel manipulator for semiconductor applications", The $8^{\text {th }}$ Mechatronics Forum International Conference, pp.1358-1366, 2002.

[4] M. Gallant and R. Boudrea, "The synthesis of planar parallel manipulators with prismatic joints for an optimal, singularity-free workspace", Journal of Robotic System, Vol.19, No.1, pp.13-24, 2002.

[5] C. Gosselin and J. Angeles, "A global performance index for the kinematic optimization of robotic manipulators", Journal of Mechanical Design, Vol.113, pp.220-226, 1991.

[6] S. Leguay-Durand and C. Reboulet, "Optimal design of a redundant spherical parallel manipulator", Robotica, Vol.15, pp.399-405, 1997.

[7] J. Ryu and J. Cha, "Optimal architecture design of parallel manipulators for best accuracy", IEEE/RSJ International Conference on Intelligent Robots and Systems, pp.1281-1286, 2001.

[8] L.W. Tsai and S. Joshi, "Kinematics and optimization of a spatial 3-UPU parallel manipulator", Journal of Mechanical Design, Vol. 122, pp.439-446, 2000. 